\title{
Environmental risk assessment of Genetically Modified Plants (GMO) - challenges and approaches
}

\author{
Justyna A. Nowakowska ${ }^{1} \bowtie$, Matgorzata Sułkowska $^{1}$, Tomasz Oszako ${ }^{2,3}$ \\ ${ }^{1}$ Forest Research Institute, Department of Silviculture and Genetics, Sękocin Stary, Braci Leśnej 3, 05-090 Raszyn, \\ Poland, phone: +4822 7150467, fax: +4822 7150313, e-mail: J.Nowakowska@ibles.waw.pl \\ ${ }^{2}$ Forest Research Institute, Department of Forest Protection, Sękocin Stary, Braci Lesnej 3, 05-090 Raszyn, Poland \\ ${ }^{3}$ European Food Safety Authority (EFSA), Via Carlo Magno 1, 43100 Parma, Italy
}

Never before in the history, the invasion of the alien pest had been so numerous, causing important financial losses, and at the same time EU countries had faced the lack of efficient tools to control them. The concern of public health became an urgent issue, livelihoods of growers are threatened, and export markets exposed at high risk, if policies for mitigating the pest impact are not being urgently modernized. Nowadays, these problems are increasing because the ability of exotic species to invade virtually all regions of the word has been accelerated by global climate change, increased international travel, and on-going difficulties of detection in early invasion stages. Consequently, invasive pest and pathogen problems have not only begun to place growers and commodity exporters in many countries at great financial risk, but have also stretched to the limits the capabilities of regional, national and international regulatory agencies for dealing with them.

Because of the ever-increasing numbers of insect pests that are invading importing and exporting countries throughout the word, a three-day minisymposium and workshop entitled "Opportunities for enhancement of integrated pest management" were held in April 1-3, 2014 at Warsaw University of Life Sciences (SGGW), involving 45 prominent entomologists, plant pathologists, invasion biologists, and policy advisers. Among different topics of a symposium, the induced plant resistance, functional biodiversity, the role of ecological infrastructures and invasive (al- ien) pests and natural enemies, as well as perspectives and bottlenecks in manipulation of insect behaviour for enhancement of integrated pest management (IPM) were discussed. The workshop dealt with implication of biodiversity in genetically modified plants, and with on-farm behaviour of European cherry fly (Rhagoletis cerasi L.) and spotted wing drosophila (Drosophila suzukii [Matsumura]).

General concept of implementation of the primary operational domains of invasive insect's policy including prevention, detection, intervention and trade was presented by James R. Carey from University of California. He highlighted four principal recommendations including the growing need for: 1) greater complementarities and integration across the main policy domains which are currently decoupled, 2) greater use of the basic principles of invasion biology to inform invasive pest policy, 3) increased use of modern science, scientific methods, and technology, particularly the use of molecular tools, mathematical models, and methods of risk management, and 4) more measured realistic approaches for intervention based on strict preconditions criteria and exit strategies.

Recently, in April 2014 the COST Action FP0905 concerning the GM forest tree species has just ended. This initiative aimed to evaluate the scientific knowledge of GM trees related to biosafety protocols between 27 involved EU countries, and 7 non-EU countries (e.g. China, New Zealand, USA). The chair of the COST 
Action, Dr Cristina Vettori from NRC, Plant Genetics Institute (Florence, Italy) stated that since far, the evaluation of the GM environmental impact has been well developed, many types of surveys have been conducted worldwide, and socio-economic risk evaluated.

The possible environmental impacts of genetically modified (GM) crops are still a controversial issue in Europe and in the world. The authorization of GMOs for the experimental release into the environment as well as for the commercial placing on the market is subject to detailed assessment and approval process. In Europe, the market commercialisation of GMO crops is carefully considered by the European Commission under the EC Regulation $N^{\circ} 1829 / 2003$ and Directive of the European Parliament and of the Council 2001/18/ EC of 12 March 2001 on the deliberate release into the environment genetically modified organisms. For enabling science-based risk assessment of GMO products, the Commission refers to the opinion of independent experts and international organisations such as the European Food Safety Authority (EFSA). The EFSA was established by the European Commission as an independent agency in 2002, in order to provide an objective scientific advice about risk assessment and risk communication of food and feed safety including animal health, and welfare and plant protection. Very soon, in 2003, the Panel of GMO was created within EFSA organisation, to provide independent scientific advice on safety of transgenic plants, as well as genetically modified animals and microorganisms, and on GM food and feed for humans, animals and environment. A crucial part of the EFSA GMO Panel's remit is the development of guidance documents to present its approach to risk evaluation and to provide notifiers with clear guidelines for the preparation and presentation of applications. The outputs of the EFSA Panel's are scientific opinions which support the European Commission and Member States when taking decisions on the authorization of GMO in the EU.

For this reason, in $9-10^{\text {th }}$ of April, 2014, the $89^{\text {th }}$ Scientific Panel on Genetically Modified Organisms recently took place in Parma (Italy), chaired by Prof. Joe Perry from Rothamsted Research (UK), the president of the GMO EFSA Panel in years 2012-2015, and concerned the authorization to release of two transgenic crop varieties: 1) the soybean MON 87769 (Application $\mathrm{N}^{\circ}$ EFSA-GMO-UK-2009-76), and 2) the oilseed rape
MON 88302 (EFSA-GMO-BE-2011-101), in accordance to the European Commission's Regulation (EC) No 1829/2003 and Directive 2001/18/EC. The panel debate was supported by transparent scientific, administrative and managerial activities, as far as the sessions were open to 41 independent observers from 16 countries, i.e. France, Italy, Belgium, UK, Denmark, Poland, Germany, Norway, Turkey, USA, Indonesia, Thailand, Laos, Malaysia, Cambodia, and India.

Debate within the EFSA GMO Panel aimed at detailed assessment of the risk of introduction of two GMO crops in the market, including: assessment of risk management, monitoring of the stability of the transgene, and the impact of human and animal health. In the case of transgenic soybean MON 87769 (EFSAGMO-UK-2009-76), environmental issues were almost negligible, because the plant genome modification involved the insertion of two genes, i.e. Pj.D6D of Primula juliae and Nc.Fad3 of Neurospora crassa, encoding proteins involved in modification of fatty acid metabolism in plants. The resulting new variety of soybean MON 87769, has higher efficiency of nutrient in produced feed. The second transgenic crop, the genetically modified oilseed rape MON 88302 (EFSAGMO-BE-2011-101), contained one "foreign" gene (CP4 epsps of Pisum sativum L.), conferring a resistance to glyphosate herbicide (e.g. Roundup ${ }^{\circledR}$, Monsanto, USA). In this case, the GMO release product exhibited higher risk on environment, mainly caused by possibility of the horizontal transfer of the transgene (from the plant to bacteria, and from plant to plant).

During debate, Prof. Joe Perry in accordance to most critical EFSA commitments, provided independent, evidence-based advice and clear communication, grounded in the most up-to-date scientific information and guided by the principles of scientific excellence, transparency and responsiveness. The scientific risk assessment conducted today in Europe needs to be explained better, more in detail, to those who are outside the process of GMO Panel guidance development.

In discussion, the topic of genetically modified tree species (e.g. transgenic Bt-poplar plantations), emphasising the need for long-term studies on the effects of the transgene (cry) on the environment was raised by $\mathrm{Dr}$ Justyna Nowakowska. EFSA regularly publishes guidelines (2006, 2011 and 2013) on GMO crop species (such as maize, potato and oilseed rape), but there is no (or 
very little) environmental impact assessment of longliving plant species (including woody species). In fact, the Bt-toxin is encoded by the transgene throughout all stages of poplar development (i.e. until flowering, that is about $10-13$ years for Populus sp.). During this time, the constitutive (continuous) expression of cry gene(s) may enhances a risk of pest-resistance against the Bttoxin(s), and the long-term effects of the product encoded by the transgene on soil microorganisms.

Such debates are particularly important in field of the genetically modified organisms where there are differing views, and where the scope of EFSA's mandates extends beyond the traditional remit of food safety to include, for example environmental risk assessment. Within the whole process of GMO acceptance to the market release, it is crucial to describe the role of new technologies, strengthen the understanding of consumer perception of risk and to build the dialogue with stakeholders. Thus, the outcome of the environmental risk assessment study allowed the identification of the possible threat issuing from GMO release, mainly consisting in: persistence of GM plants including plant-to-plant gene transfer, plant-to-microorganism vertical transfer, interaction between GM plants with target organisms (pathogens or pests), impact of the cultivation on environment, impact on biochemical processes, and effect on human and animal health.

Further interest on GM-plants will be focused on environmental impact of perennial plants, including forest tree species, as well as on relevance of modelling for assessing spatial and temporal effects of GMOs in general. 